\title{
Safety and feasibility of physiotherapy in ICU-admitted severe COVID-19 patients: an observational study
}

\author{
Veronica Rossi ${ }^{1,2^{*}}$, Martina Santambrogio ${ }^{2 *}$, Cesare Del Monaco ${ }^{1,2}$, Mariangela Retucci ${ }^{1,2}$, \\ Serena Tammaro ${ }^{1,2}$, Clara Ceruti ${ }^{1,2}$, Laura Saderi ${ }^{3}$, Stefano Aliberti ${ }^{4,5}$, Emilia Privitera $^{1,2}$, \\ Giacomo Grasselli, ${ }^{6,7}$, Giovanni Sotgiu ${ }^{3}$, Francesco Blasi ${ }^{2,6}$
}

${ }^{1}$ Health Professions Department Unit, Fondazione IRCCS Ca' Granda Ospedale Maggiore Policlinico, Milan; ${ }^{2}$ Respiratory Unit and Cystic Fibrosis Adult Center, Fondazione IRCCS Ca' Granda Ospedale Maggiore Policlinico, Milan; ${ }^{3}$ Department of Medical, Surgical and Experimental Sciences, University of Sassari; ${ }^{4}$ Department of

Biomedical Sciences, Humanitas University, Pieve Emanuele, Milan; ${ }^{5}$ Respiratory Unit, IRCCS Humanitas Research Hospital, Rozzano, Milan; ${ }^{6}$ Department of

Correspondence: Emilia Privitera, PT, MSc, Fondazione IRCCS Ca' Granda Ospedale Maggiore Policlinico, Health Professions Department Unit, Via Francesco Sforza 28, 20122 Milano, Italy. Tel. 0039 3385670909. E-mail: emilia.prvitera@policlinico.mi.it

Key words: Early rehabilitation; severe COVID-19; critical illness; physiotherapy; safety; feasibility.

Acknowledgements: The authors would like to acknowledge Filippo Cuccarini for the support, Stefano Zampese for critical reading of the manuscript and all the physicians, physiotherapists and nurses of our Hospital.

Contributions: VR, MS, CD, MR, ST and GG literature search; VR, MS, CD, ST, CC and MR data collection; VR, MS, CD, MR, SA, EP and GS and FB study design; VR, MS, GS and LS analysis of data; VR, MS, MR, GG, GS and FB manuscript preparation; VR, MS, CD, MR, ST, CC, LS, SA, EP, GG, GS and FB review of manuscript. All the authors have read and approved the final version of the manuscript and agreed to be accountable for all aspects of the work.

Funding: This research did not receive any specific grant from funding agencies in the public, commercial, or not-for-profit sectors.

Conflict of interest: Nothing to disclose.

Ethical approval and consent to participate: Data were collected and analysed only by researchers, providing full confidentiality and anonymity. The study was approved by the Ethical Committee on May 21st 2020 (IRB: 445_2020). Informed consent was waived due to the retrospective nature of the study. This study complies with the Declaration of Helsinki.

Received for publication: 14 September 2021.

Accepted for publication: 14 January 2022.

Publisher's note: All claims expressed in this article are solely those of the authors and do not necessarily represent those of their affiliated organizations, or those of the publisher, the editors and the reviewers. Any product that may be evaluated in this article or claim that may be made by its manufacturer is not guaranteed or endorsed by the publisher.

COpyright: the Author(s), 2022

Licensee PAGEPress, Italy

Monaldi Archives for Chest Disease 2022; 92:2087

doi: 10.4081/monaldi.2022.2087

This article is distributed under the terms of the Creative Commons Attribution-NonCommercial International License (CC BY-NC 4.0) which permits any noncommercial use, distribution, and reproduction in any medium, provided the original author(s) and source are credited.
Pathophysiology and Transplantation, University of Milan; ${ }^{7}$ Department of Anesthesia, Critical Care and Emergency, Fondazione IRCCS Ca' Granda - Ospedale Maggiore Policlinico, Milan, Italy

\section{Introduction}

In February 2020 the first case of severe acute respiratory syndrome coronavirus 2 (SARS-CoV-2) was notified in Italy and the 
number of positive individuals, needing intensive care, rapidly increased $[1,2]$. SARS-CoV-2 infected individuals showed different clinical manifestations, ranging from a- or pauci-symptomatic condition to critical illness, with respiratory failure, requiring intensive care unit (ICU) admission [3]. A high proportion of ICU patients need invasive mechanical ventilation (IMV) and about $50 \%$ of the ICU-admitted patients die [4-7]. The median age of ICU patients with coronavirus disease (COVID-19) was $<65$ years and the mortality in patients aged $<63$ years ranges from 15 to $20 \%$ $[1,3,5,7]$.

Early rehabilitation is safe and effective in critically ill patients and, in patients with acute respiratory distress syndrome (ARDS), it helps to reduce the functional impairment due to the prolonged stay in ICU $[3,8,9]$. Preliminary data suggest the implementation of early and active mobilization programs, as well as airway clearance, for patients with severe forms of COVID-19 [10-15].

The trend of infections in Italy showed a slow decrease until July 2020 and since August 2020 the number of new positive cases progressively has increased [16]. Several precautions, including the use of adequate personal protection equipment (PPE), were recommended during treatment of COVID-19 patients and physiotherapists modified their interventions accordingly [11]. Unfortunately, high quality data on safe and effective physiotherapy interventions are missing.

The aim of the present study was to assess safety and feasibility of early physiotherapy in severe COVID-19 ICU-admitted patients. The path and timing of physiotherapy during ICU stay until hospital discharge were described. The interventions most frequently delivered in each hospital setting were identified, along with the time taken to first mobilize, stand and walk. Functional and clinical status of patients were evaluated at the fist physiotherapy assessment and at hospital discharge.

\section{Methods}

\section{Study design and setting}

An observational, retrospective, monocentric study was performed in a large teaching hospital in Milan (Italy) to examine safety and feasibility of early physiotherapy in critically ill COVID-19 patients. From February $1^{\text {st }}, 3$ ICUs, 2 intermediate care units (IMCU) and 4 general ward units were dedicated to the treatment of COVID-19 patients. In February 2020, more than 100 patients were admitted to ICU and 300 patients to other COVID19 units. Among the 24 physiotherapists from different units assigned to acute COVID-19 wards, 10 have a postgraduate degree in cardio-pulmonary and critical care physiotherapy or had $>5$ years' experience in the respiratory field [17]. Day shifts were rescheduled to ensure the presence of physiotherapists from $8 \mathrm{am}$ to $8 \mathrm{pm}, 7 / 7$ days and PPE was adopted as required [2,18]. Among the 24 physiotherapists working at the COVID-units, no SARS-CoV-2 positive cases were found.

\section{Study population}

Patients with a laboratory-confirmed SARS-CoV-2 infection, admitted to our hospital from March $1^{\text {st }} 2020$ to May $31^{\text {st }} 2020$ were consecutively recruited.

The inclusion criteria were: i) age $\geq 18$ years; ii) laboratoryconfirmed COVID-19 diagnosis; iii) admission to ICU for acute respiratory distress syndrome; iv) intubated on invasive ventilation for $>24$ hours; v) treatment by respiratory physiotherapist during the ICU stay.

Patients with at least one of the following criteria were excluded from the study: i) cognitive impairment before the diagnosis of SARS-CoV-2 infection; ii) neuromuscular, orthopaedic, or any other disease hindering ambulation.

\section{Procedures and interventions}

Two investigators retrospectively screened medical records and the data on physiotherapy were retrieved. Since there are no respiratory physiotherapists in Italy, physiotherapy interventions included respiratory and rehabilitation activities. Each patient in ICU and IMCU received 2 physiotherapy sessions of at least 40 minutes every day. During general ward stay one physiotherapy session per day was delivered and autonomous work was assigned. In every physiotherapy session many activities were included.

COVID-19 patients were evaluated and treated by physiotherapists from ICU until hospital discharge as described in Table 1 .

\section{Outcomes}

Primary outcomes were: i) number and type of physiotherapy treatments performed during hospitalisation; ii) number of physiotherapy-related adverse events (AE).

Secondary outcome was the assessment of functional and clinical status of patients at hospital discharge.

The following variables were recorded: i) physiotherapy treatments performed during hospitalisation; ii) first time sitting out of bed, standing and walking; iii) six-minute walking test (6MWT) and 1-minute sit-to-stand test (1m-STST) [19,20]; iv) muscle strength measured by Medical Research Council Sum Score (MRC-SS) of upper and lower extremities [21]; v) functional independence in activities of daily living (ADL) assessed by the Barthel Index for ADL [22]; vi) length of ICU and hospital stay; vii) duration of IMV; viii) discharges at home, to in-patient rehabilitation or transferred to other hospital; ix) in hospital deaths for any cause; $x$ ) Manchester mobility score (MMS) [23].

\section{Data analysis}

Categorical variables were described as number and percentages, whereas continuous variables were summarized with means (standard deviations, SD) or medians (interquartile ranges, IQR) depending on their parametric distribution. Chi-squared test or Fisher exact tests were used to compare comorbidities between patients exposed to non-invasive mechanical ventilation (NIMV), continuous positive airway pressure (CPAP), and high flow nasal oxygen (HFNO). In-between group comparisons of quantitative variables were performed with Kruskal-Wallis test. A two-tailed $\mathrm{p}$ value $\leq 0.05$ was considered statistically significant. All analyses were carried out with STATA version 16 (StatsCorp, Texas, US).

\section{Ethical considerations}

Data were collected and analysed only by researchers, providing full confidentiality and anonymity. The study was approved by the Ethical Committee on May 21 2020 (IRB: 445_2020). Informed consent was waived due to the retrospective nature of the study. This study complies with the Declaration of Helsinki.

\section{Trial registration}

This study was registered on clinicaltrials.gov (July $1^{\text {st }} 2020$; NCT04459819). 


\section{Results}

\section{Patients' demographic and clinical characteristics}

Eighty-four severe COVID-19 patients were enrolled. Demographic and clinical characteristics of the population and ICU therapies are reported in Table 2 . All patients were mechanically ventilated and the median (IQR) duration of IMV was 16.5 (10.5$31)$ days. Before intubation, $64(76.2 \%)$ patients were treated with CPAP, 4 (4.8\%) with NIMV, and $3(3.6 \%)$ with HFNO. After the removal of the endotracheal tube, $10.7 \%$ were treated with NIMV and HFNO while $59.5 \%$ with CPAP. Tracheostomy was performed in 21 patients $(25.0 \%)$, after median (IQR) 25 (19.75-30.75) days from intubation. Transtracheal open ventilation (TOV) was used with $33.3 \%$ of the patients who had tracheostomy (Table 2)

The majority of patients were transferred to ICU from other COVID wards, mainly IMCU of the same hospital (36.9\%) and ICU of other hospitals (25.0\%). Individuals that needed an upgrade from IMCU of our hospital to ICU, showed a median (IQR) IMCU stay of 4 (3-6) days and already started physiotherapy treatment in this ward (Table 2).

The median (IQR) hospital stay was 40.5 (29-53) days, with almost $50 \%$ of the hospital stay spent in ICU (Table 2). Figure S1 describes distribution and movements of all patients from admission to discharge.

\section{Safety of physiotherapy in severe COVID-19 patients}

During physiotherapy interventions, $32(0.58 \%)$ adverse

Table 1. Patient assessment and treatment. COVID-19 patient evaluation and physiotherapy interventions performed in ICU, IMCU and general ward are described here.

$\begin{array}{ll}\text { Setting/patient status } & \text { Assessment } \\ \text { In ICU } & \bullet \text { Lung volumes } \\ \text { Patient sedated, intubated } & \bullet \text { Cardiopulmonary function } \\ \text { and on IMV } & \bullet \text { Respiratory and peripheral muscle evaluation } \\ & \bullet \text { Respiratory reserve and hemodynamic response } \\ & \text { during patient positioning and early mobilization } \\ & \text { - Agitation and sedation level }\end{array}$

In ICU

Patient weaning form IMV

In IMCU and general ward

Tracheostomy management tionality during pressure support ventilation

- Respiratory and peripheral muscle evaluation

Response during patient positioning and early mobilization

- Ability to perform bed mobility activities

- Ability to reach and maintain sitting and vertical position while receiving minimal or no ventilator support
- Lung volumes

- Cardiopulmonary function

- Respiratory and peripheral muscle evaluation

- Oxygen support need

- Ability to reach and maintain sitting and vertical position

- Mobility level

- Balance and gait assessment

- Swallowing assessment
- Cough efficacy assessment

- Swallowing assessment

- Humidification need

Treatment

- Respiratory and peripheral muscles training

- Reaching and maintaining lateral, sitting, and vertical positions

- Modification of IMV settings during mobilization and airway clearance according to the respiratory need of the patient

- Active mobilization in bed and ADL training to promote functional status and independence in basic activities of daily living (e.g., grabbing objects, bed mobility)

- Airway clearance strategies

- Lung expansion techniques (e.g., deep breathing exercises)

- Aerosol administration

- Management of oxygen support

- Complete assessment of respiratory mechanics and func- $\bullet$ Assist IMV weaning

- Support patient with non-invasive respiratory support after extubation

- Airway clearance strategies

- Spontaneous breathing trials

- Management of CPAP and NIMV cycles

- Bed mobility activities

- Active exercises

- Strengthening exercises

- ADL training

- Weight shifting exercises

- Balance training

- Transfer training

- Pre-gait exercises and ambulation

- Endurance training

- Autonomous exercises prescribed

- Spontaneous breathing trials

- TOV

- Tracheo-suction

- Tracheo weaning

- Airway clearance strategies

- Phonatory re-education

ADL, activities of daily living; CPAP, continuous positive airway pressure; ICU, intensive care unit; IMCU intermediate care unit; IMV, invasive mechanical ventilation; NIMV, non-invasive mechanical ventilation; TOV, transtracheal open ventilation. 
events occurred, 5 in ICU, 27 in IMCU and none in the general ward. No unplanned extubations and arterial catheter removal were reported. Physiotherapy-related AE were mainly tachycardia, hypotension, desaturation to $\mathrm{SpO}_{2}<80 \%$, falls and hypertension (Table 3). In IMCU, most AE occurred during patient mobilization and walking whereas in ICU, AE occurred during patient positioning and airway clearance. Five $(5.6 \%)$ patients were readmitted to the ICU for deterioration of respiratory conditions.

Table 2. Demographic, clinical and admission characteristics of the cohort.

\begin{tabular}{lc}
\hline Patients' demographics and symptoms at ICU admission \\
Age, mean (SD) years & $56.0(10.5)$ \\
Males, $\mathrm{n}(\%)$ & $63 / 84(75.0)$ \\
\hline BMI, median (IQR) kg/m2 & $27.0(24.7-29.7)$ \\
Comorbidities & \\
Respiratory disease, $\mathrm{n}(\%)$ & $6 / 84(7.1)$ \\
Hypertension, n (\%) & $16 / 84(19.0)$ \\
Diabetes, $\mathrm{n}(\%)$ & $6 / 84(7.1)$ \\
Anxiety/depression, $\mathrm{n}(\%)$ & $8 / 84(9.5)$ \\
Cardiovascular disease, $\mathrm{n}(\%)$ & $11 / 84(13.1)$ \\
Neuropathy, $\mathrm{n}(\%)$ & $4 / 84(4.7)$ \\
Obesity, $\mathrm{n}(\%)$ & $12 / 84(14.2)$ \\
Neoplastic disease, $\mathrm{n}(\%)$ & $1 / 84(1.2)$ \\
Other comorbidities, $\mathrm{n}(\%)$ & $15 / 84(17.8)$ \\
No comorbidities, $\mathrm{n}(\%)$ & $21 / 84(25.0)$
\end{tabular}

Symptoms pre-diagnosis

\begin{tabular}{lc} 
Lethargy, $\mathrm{n}(\%)$ & $27 / 84(32.1)$ \\
Fever, $\mathrm{n}(\%)$ & $77 / 84(91.7)$ \\
Dyspnea, $\mathrm{n}(\%)$ & $56 / 84(66.7)$ \\
Cough, $\mathrm{n}(\%)$ & $41 / 84(48.8)$ \\
Access from & \\
ICU of other hospital, $\mathrm{n}(\%)$ & $21 / 84(25.0)$ \\
Stay in ICU of other hospitals, median days (IQR) & $4(3-8.25)$ \\
Not ICU ward of other hospital, n (\%) & $7 / 84(8.3)$ \\
COVID-IMCU of same hospital, $\mathrm{n}(\%)$ & $31 / 84(36.9)$ \\
Stay in COVID-IMCU, median days (IQR) & $4(3-6)$ \\
ED of same hospital, $\mathrm{n}(\%)$ & $22 / 84(26.2)$ \\
COVID-general ward of same hospital, n (\%) & $2 / 84(2.4)$ \\
Stay in COVID-general ward, median days (IQR) & $5(4-5.5)$ \\
Not COVID-general ward of same hospital, $\mathrm{n}(\%)$ & $1 / 84(1.2)$ \\
\hline ICU therapy &
\end{tabular}

\begin{tabular}{lc} 
ECMO, n (\%) & $2 / 84(2.4)$ \\
Continuous renal replacement therapy, n (\%) & $7 / 84(8.3)$ \\
\hline Antiviral therapy, n (\%) & $46 / 84(54.8)$ \\
Hydroxychloroquine, n (\%) & $79 / 84(94.1)$ \\
\hline Ventilation and respiratory support & \\
IMV duration, median (IQR) days & $16.5(10.5-31)$ \\
Tracheotomy packaging, n (\%) & $21 / 84(25.0)$ \\
Hospital stay &
\end{tabular}

Duration of hospitalization, median (IQR) days

$40.5(29-53)$

ICU length of stay, median (IQR) days

$17(12.5-29.5)$

IMCU length of stay, median (IQR) days *

$16(9-25)$

General ward length of stay, median (IQR) days **
BMI, body mass index; COVID-19, coronavius disease 2019; CPAP, continuous positive airway pressure; ECMO, extracorporeal membrane oxygenation; ED, emergency department; HFO2, high flow oxygen; ICU, intensive care unit; IMCU, intermediate care unit; IMV, invasive mechanical ventilation; NIMV, noninvasive mechanical ventilation; PEEP, positive end-expiratory pressure; PS, pressure support, TOV, transtracheal open ventilation; *IMCU length of stay was calculated among the 77 patients ( $92 \%$ of overall enrolled) that were admitted to IMCU; ${ }^{* *}$ general ward length of stay was calculated among the 24 ( $29 \%$ of overall enrolled) patients that were admitted to general ward.
Seen the contagiousness of SARS-CoV-2, also physiotherapists' safety should be taken into account; among the physiotherapists working at the COVID wards, no SARS-CoV-2 positive cases were found during the duration of the whole study.

\section{Physiotherapy activities in severe COVID-19 patients}

The median (IQR) number of physiotherapy entries registered during the hospital stay was 60.5 (36-93). As soon as sedation was reduced and clinical conditions were stable, physiotherapists started their activity at the ICU and treatment continued in IMCU and general ward, until hospital discharge. At the first physiotherapy assessment all patients presented Glasgow coma scale (GCS) $\geq 7$. Patients' mobility level was measured by the MMS and $12.0 \%$ of them were able to sit on the edge of bed, while the remaining $88.0 \%$ received in bed interventions during the first physiotherapy treatment (Table S1).

Patient positioning into prone or lateral position to improve gas exchange was performed on sedated and intubated patients in ICU. Passive mobilization was mainly performed during the early phase of the rehabilitation, whereas active mobilization and muscle strengthening exercises were recommended as soon as patients could perform them, with a particular focus on independency in ADL. In the ICU, physiotherapists were involved in lung expansion techniques and airway clearance (Figure 1A). To investigate whether comorbidities could predict the administration of a specific non-invasive support after extubation, patients with comorbidities exposed to different non-invasive support after orotracheal tube removal were compared and no differences were found.

During the first assessment in IMCU the mobility level of patients increased as compared to first ICU evaluation (Table S1). In IMCU the most prevalent respiratory activities were oxygen management and weaning, followed by treatment with CPAP or NIMV, swallowing assessment and lung expansion. Pronation and patient positioning into lateral position were performed in awake patients during CPAP or high flow oxygen treatment in IMCU (Figure 1B) [24].

The number of entries involving ADL training and transfer/mobilization increased while patients moved from ICU to intermediate and low care settings (Figure 1).

The median (IQR) time interval from patient intubation to the first physiotherapy treatment was 13 (9.0-21.5) days (Table 4). The time to first treatment significantly increases with patients' age $(p=0.03)$ and duration of IMV $(p<0.0001)$, whereas it is not influenced by number of comorbidities $(p=0.54)$, continuous renal replacement therapy $(\mathrm{p}=0.07)$, use of extracorporeal membrane oxygenation (ECMO) $(\mathrm{p}=0.79)$ and body mass index (BMI; $\mathrm{p}=0.30$; Table S2). Similar results were found for time interval from intubation to sitting out of bed, standing and walking.

A tracheostomy was performed in 21 patients, after prolonged intubation and sedation. In patients that underwent tracheostomy the first physiotherapy treatment and mobility activities were delayed $(p<0.0001)$ and the median number of activities performed on patients with tracheostomy was higher than patients without tracheostomy ( $\mathrm{p}=0.0006$; Table S2).

\section{Patient outcomes}

Six $(7.1 \%)$ patients died, 2 of which with tracheostomy. Five died in ICU on IMV (mean hospital stay 28.5 days). Medical records were analysed to extract outcome measures at ICU and hospital discharge and results are reported in Figure S2. The mean (SD) $\mathrm{PaO}_{2} / \mathrm{FiO}_{2}$ was 368.8 (66.3). At hospital discharge, $85.7 \%$ of patients reached the highest rehabilitation mobility level (mobilizing $>30 \mathrm{~m}$ ) at the MMS (Table S1). No differences were found in 
the performance at the 6MWT and 1m-STST in the subgroups of patients which had tracheostomy as compared with patients that had not, whereas a difference was found in hospital discharge destination, with a larger proportion of tracheo-patients discharged to in-patient rehabilitation with lower scores at Barthel Index for ADL (Figure S2).

No significant differences were found comparing outcome measures at hospital discharge in patients with $\mathrm{BMI} \geq 25$ and $<25$ (Figure S2).

\section{Discussion}

This study describes the physiotherapy-related interventions delivered to severe COVID-19 patients during their ICU stay until

A

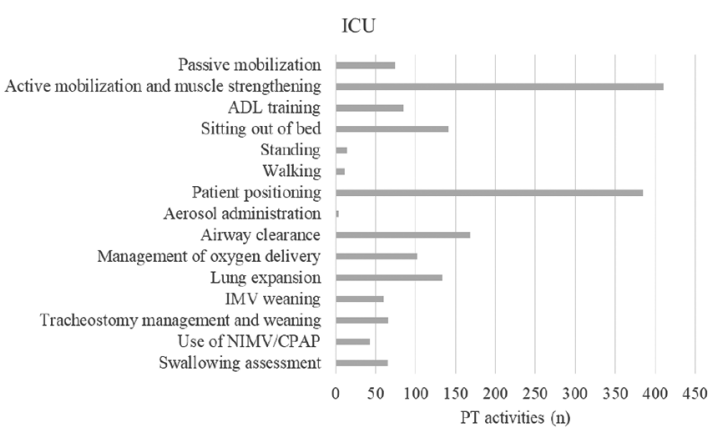

B

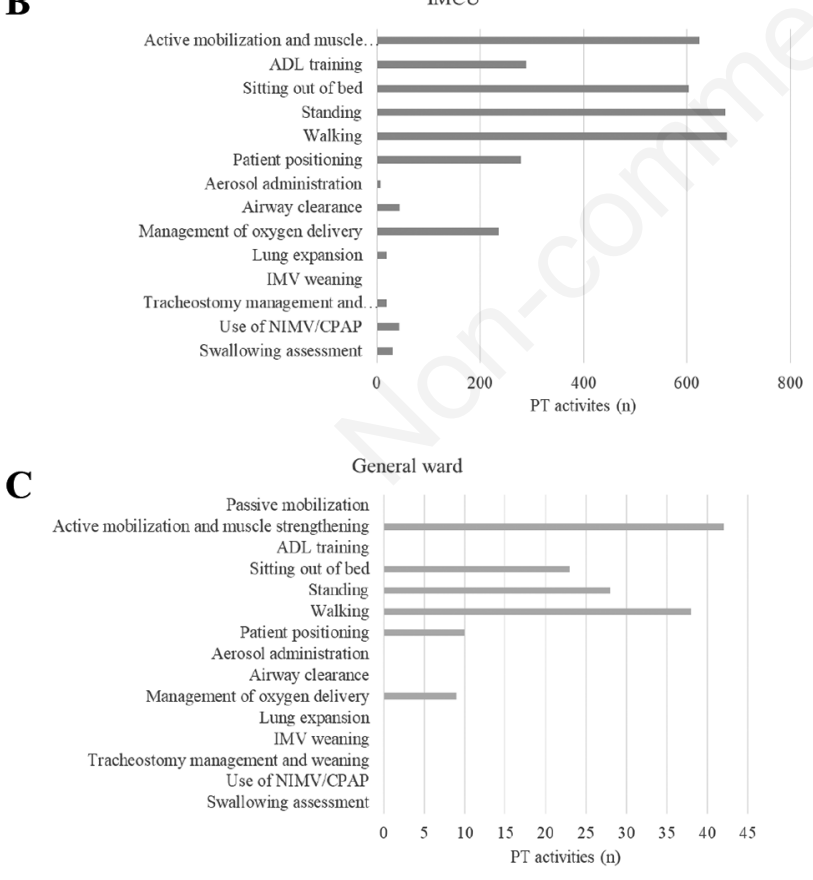

Figure 1. Physiotherapy intervention delivered during hospital stay. A) Activities delivered by physiotherapists during ICU stay B) Activities delivered by physiotherapists during IMCU stay C) Activities delivered by physiotherapists during general ward stay Abbreviations: ADL, activities of daily living; ICU, intensive care unit; IMCU, intermediate care unit; IMV, invasive mechanical ventilation; NIMV, non-invasive mechanical ventilation; CPAP, continuous positive end-expiratory pressure. the hospital discharge in a large teaching hospital in Milan, Italy. These data summarise the very early experience of our physiotherapy team facing the COVID-19 pandemic and have been useful to identify the most used and safe interventions for this critical population.

Although previous works clearly demonstrated the importance of early physiotherapy in critically ill patients, some studies showed institutional, clinical and logistic barriers to the implementation of early rehabilitation programs [25-27]. In this context of pandemic and emergency, it was a possible scenario that physiotherapy was not considered a priority, especially in ICUs [28]; thus, it appeared very important to verify the feasibility and safety of physiotherapy and rehabilitation in these new severe infectious patients.

Early mobilization and respiratory treatments were feasible and safe in this patient group. In our study population, physiotherapy interventions were performed from ICU awakening until hospital discharge. We found that the first interventions occurred around the extubation period (13 days post intubation), in line with recent data from McWilliams et al. that investigated the levels of mobility and rehabilitation in a UK population of ICU-admitted COVID-19 patients [15]. During the rehabilitation, physiotherapy was performed 7 days per week, with individualised interventions. Organizing physiotherapists during the pandemic was very challenging, due to the high working demands which required the reorganization of shifts and working teams, the effort to reduce to a minimum the risk of virus spreading during aerosol generating procedures, the space limitations due to isolation of infected patients and the number of PPE required to work safely. Despite all of these challenging conditions, none of the physiotherapists working inside COVID-units was infected and a few minor adverse events occurred during physiotherapy, mainly in intermediate care units during patient mobilization, transfer and walking.

The physiotherapy for severe COVID-19 patients did not differ if compared with that administrated to other critically ill subjects, with the only exception for the organizational and logistic barriers related to the contagiousness of the infection. Severe COVID-19 patients showed prolonged IMV duration and ICU stay, leading in the majority of cases to ICU acquired weakness and long-term consequences, such as muscle weakness and fatigue $[15,29]$. In the present study, we provided information on IMV, date of intubation, non-invasive ventilation support used before intubation. No differences were found in patients referred from different hospitals.

In our patient population, BMI did not affect the timing of first physiotherapy treatment, while other authors showed that the time to initial mobilization increased significantly with BMI [15]. This could maybe be linked to the specific population analysed. In our study, only patients that received physiotherapy in ICU were included and at the first physiotherapy treatment $12.0 \%$ of patients was able to sit on the edge of the bed. On the contrary, ICUacquired weakness was present for all patients on awakening in the population studied by Mc Williams et al. [15].

A functional and respiratory patient assessment was obtained at hospital discharge and results showed that patients' strength, function and independence in daily life improved during the hospital stay. Patients achieved a mean walking distance and sit-tostand repetitions of 247.8 meters and 15.6 at hospital discharge. Available literature data are very heterogeneous and show that in critically ill patients 6 -minutes walking distance ranges from $<35$ to $>250$ meters, with lower distances often achieved by ARDS survivors $[8,30,31]$.

Moreover, at hospital discharge $>80 \%$ of patients showed a 
MRC-SS score $>48$, with a progressive increased muscle strength from ICU stay to hospital discharge and $62.1 \%$ were functionally independent at hospital discharge, with a Barthel Index for ADL score $>90$. A recent work demonstrated low physical functioning and poor performance in ADLs in COVID-19 patients at hospital discharge from a rehabilitation hospital admitting post-acute SARS-CoV-2 positive subjects [32]. Compared to this study, although $100 \%$ of the patients enrolled in our study were intubated and mechanically ventilated and only $11.7 \%$ of patients form the study of Belli et al. received IMV, we observed a lower proportion of subjects with abnormal performance of activities of daily living at hospital discharge (7.1\% patients scored $<60$ at Barthel Index in our study vs $47.5 \%$ in Belli et al. work). We can speculate that a structured programme of early physiotherapy interventions can increase functional outcomes, but this could be linked also to our specific population of severe COVID-19 patients that is younger than the study population of Belli et al. [mean (SD) age 56 (10.5) years VS. 74.3 (12.7) years]. As most of our patients fall in the working age, the implications of a proper functional recovery in working capacity must also be accounted.

$57.1 \%$ of our patients returned to their accommodation, whereas $34.5 \%$ were referred to rehabilitation centres and $7.1 \%$ died. Similar results were obtained by McWilliams et al. [15]. Although discharge destination from hospital is multifactorial, independence of home life before the admission, mean age, early start of standing and walking, functional independence in ADL, and the nature of pandemic, could be identified as factors strongly related to discharge to home. These data altogether are quite relevant as they suggest that early rehabilitation can increase functional outcomes that facilitate home discharge, with a reduction of costs but also an important impact on the rehabilitative inpatient structures that have limited resources and should face increasing bed demand during the pandemic. This rate of home discharged patients is in accordance with previous works on patients treated in ICU [33].

In our hospital, extubation failure, difficult weaning and inadequate cough strength were the most common reasons to insert the tracheostomy. Patients with tracheostomy showed a delay in the first physiotherapy interventions and in reaching functional and mobility step, as first time sitting out of bed, standing and walking. Data could highlight that our medical approach potentially affected the timing of the first physiotherapy treatment. Interestingly, when discharged form hospital, these patients obtained similar results than patients without tracheostomy at the 6MWT and 1m-STST, but lower scores of independence in ADL and a lower rate of patients discharged home.

This study has some limitations. It is an observational, retrospective, single-centre study which was not planned to compare the results with those of a control group not exposed to physiotherapy; thus, no support to the efficacy of respiratory physio-

Table 3. Physiotherapy-related adverse events, reported during physiotherapy treatments.

\begin{tabular}{|c|c|c|c|}
\hline Adverse events (n) & ICU & IMCU & General ward \\
\hline Unplanned extubation & 0 & 0 & 0 \\
\hline Accidental arterial catheter removal & 0 & 0 & 0 \\
\hline Accidental feeding tube removal & 1 & 1 & 0 \\
\hline Asynchrony with mechanical ventilation & 1 & 0 & 0 \\
\hline Uncontrolled arrhythmia & 0 & 1 & 0 \\
\hline Tachycardia & 0 & 5 & 0 \\
\hline Atrial fibrillation & 1 & 2 & 0 \\
\hline Desaturation to $\mathrm{SpO}_{2}<80 \%$ & 0 & 4 & 0 \\
\hline Hypotension & 1 & 5 & 0 \\
\hline Hypertension & 1 & 3 & 0 \\
\hline Falls to knee or ground & 0 & 3 & 0 \\
\hline Other & 0 & 3 & 0 \\
\hline
\end{tabular}

ICU, intensive care unit; IMCU, intermediate care unit.

Table 4. Physiotherapy timing and details.

\begin{tabular}{lc}
\hline Variables & Total \\
Physiotherapy activities per patient during the whole hospitalization, median (IQR) & $60.5(36-93)$ \\
Time from patient's intubation to the first physiotherapy treatment, days median (IQR) & $13(9.0-21.5)$ \\
\hline Time from patient's intubation to sitting out of bed, days median (IQR) & $22(13-32)$ \\
Patients orally intubated sitting out of bed, n (\%) & $3 / 84(3.5)$ \\
\hline Patients sitting out of bed in ICU, n (\%) & $43 / 84(51.2)$ \\
Time from patient's intubation to standing, days median (IQR) & $25(15-34)$ \\
\hline Patients standing in ICU, n (\%) & $14 / 84(16.6)$ \\
Time from patient's intubation to walking, days median (IQR) & $27(16-41)$ \\
\hline Patients walking in ICU, $n(\%)$ & $5 / 84(6.0)$ \\
\hline
\end{tabular}

ICU, intensive care unit. 
therapy in severe COVID-19 patients in comparison with other therapies can be provided. Moreover, due to the nature of the study and the high number of critically ill patients admitted to our hospital during the pandemic, some patients were discharged from the hospital without a predefined assessment of the outcomes. Furthermore, COVID-19 is a novel disease and long-term follow up is needed to better evaluate the advantages of physiotherapy interventions.

\section{Conclusions}

In severe ICU-admitted COVID-19 patients, early rehabilitation is feasible and safe for patients and physiotherapists. Data about the effectiveness of physiotherapy interventions in the treatment of patients with COVID-19 is still limited.

\section{References}

1 Grasselli G, Pesenti A, Cecconi M. Critical care utilization for the COVID-19 outbreak in Lombardy, Italy: Early experience and forecast during an emergency response. JAMA 2020;323:1545-46.

2 World Health Organization. Clinical management of severe acute respiratory infection (SARI) when COVID-19 disease is suspected: interim guidance, 2020. Available from: https://apps.who.int/iris/handle/10665/331446

3 Wiersinga WJ, Rhodes A, Cheng AC, et al. Pathophysiology, transmission, diagnosis, and treatment of Coronavirus Disease 2019 (COVID-19): A review. JAMA 2020;324:782-93.

4 Bhatraju PK, Ghassemieh BJ, Nichols M, et al. Covid-19 in critically ill patients in the Seattle region - case series. N Engl J Med 2020;382:2012-22.

5 Grasselli G, Greco M, Zanella A, et al. COVID-19 Lombardy ICU network. Risk factors associated with mortality among patients with COVID-19 in intensive care units in Lombardy, Italy. JAMA Intern Med 2020;180:1345-55.

6 Yang X, Yu Y, Xu J, et al. Clinical course and outcomes of critically ill patients with SARS-CoV-2 pneumonia in Wuhan, China: a single-centered, retrospective, observational study. Lancet Respir Med 2020;8:475-81.

7 CDC COVID-19 Response Team. Severe outcomes among patients with Coronavirus Disease 2019 (COVID-19) - United States, February 12-March 16, 2020. MMWR Morb Mortal Wkly Rep 2020;69:343-46.

8 Schweickert WD, Pohlman MC, Pohlman AS, et al. Early physical and occupational therapy in mechanically ventilated, critically ill patients: a randomised controlled trial. Lancet 2009;373:1874-82.

9 Kim RY, Murphy TE, Doyle M, et al. Factors associated with discharge home among medical ICU patients in an early mobilization program. Crit Care Explor 2019;1:e0060.

10 Righetti RF, Onoue MA, Politi FVA, et al. Physiotherapy care of patients with Coronavirus Disease 2019 (COVID-19) - a Brazilian experience. Clinics (Sao Paulo) 2020;75:e2017.

11 Lazzeri M, Lanza A, Bellini R, et al. Respiratory physiotherapy in patients with COVID-19 infection in acute setting: a Position Paper of the Italian Association of Respiratory Physiotherapists (ARIR). Monaldi Arch Chest Dis 2020;90:1285.
12 Vitacca M, Carone M, Clini EM, et al. Joint Statement on the role of respiratory rehabilitation in the COVID-19 crisis: The Italian Position Paper. Respiration 2020;99:493-99.

13 Thomas P, Baldwin C, Bissett B, et al. Physiotherapy management for COVID-19 in the acute hospital setting: clinical practice recommendations. J Physiother 2020; 66:73-82.

14 Zhao HM, Xie YX, Wang C; Recommendations for respiratory rehabilitation in adults with coronavirus disease 2019. Chin Med J 2020;133:1595-602.

15 McWilliams D, Weblin J, Hodson J, et al. Rehabilitation levels in patients with COVID-19 admitted to intensive care requiring invasive ventilation. An observational study. Ann Am Thorac Soc 2021;18:122-9.

16 Armocida B, Formenti B, Ussai S, et al. The Italian health system and the COVID-19 challenge. Lancet Public Health 2020;5:e253.

17 Gambazza S. Respiratory physiotherapy education in Italy: per aspera ad astra. Breathe 2018;14:250-2.

18 Aliberti S, Amati F, Pappalettera M, et al. COVID-19 multidisciplinary high dependency unit: the Milan model. Respir Res 2020;21:260.

19 Bohannon RW, Crouch R. 1-Minute Sit-to-Stand Test: systematic review of procedures, performance, and clinimetric properties. J Cardiopulm Rehabil Prev 2019;39:2-8.

20 Holland AE, Spruit MA, Troosters T, et al. An official European Respiratory Society/American Thoracic Society technical standard: field walking tests in chronic respiratory disease. Eur Respir J 2014;44:1428-46.

21 Stevens RD, Marshall SA, Cornblath DR, et al. A framework for diagnosing and classifying intensive care unit-acquired weakness. Crit Care Med 2009;37:S299-308.

22 Mahoney FI, Barthel DW. Functional evaluation: the Barthel Index. Md State Med J 1965;14:61-5.

23 McWilliams D, Atkins G, Hodson J, et al. Feasibility and reliability of the Manchester Mobility Score as a measure of physical function within the Intensive Care Unit. ACPRC J 2015;48:26-33.

24 Retucci M, Aliberti S, Ceruti C, et al. Prone and lateral positioning in spontaneously breathing patients with COVID-19 pneumonia undergoing noninvasive helmet CPAP treatment. Chest 2020;158: 2431-35.

25 Nydahl P, Ruhl AP, Bartoszek G, et al. Early mobilization of mechanically ventilated patients: a 1-day point-prevalence study in Germany. Crit Care Med 2014;42:1178-86.

26 Koo KK, Choong K, Cook DJ, et al. Early mobilization of critically ill adults: a survey of knowledge, perceptions and practices of Canadian physicians and physiotherapists. CMAJ Open 2016;4:E448-54.

27 Harrold ME, Salisbury LG, Webb SA, Allison GT. Early mobilisation in intensive care units in Australia and Scotland: a prospective, observational cohort study examining mobilisation practises and barriers. Crit Care 2015;19:336.

28 Privitera E, D’Abrosca F, Gaudiello G, et al. Physiotherapist involvement in the pandemic era: a Lombardy region survey. Monaldi Arch Chest Dis 2021;91:1762.

29 Huang C, Huang L, Wang Y, et al. 6-month consequences of COVID-19 in patients discharged from hospital: a cohort study. Lancet 2021;397:220-32.

30 Parry SM, Nalamalapu SR, Nunna K, et al. Six-minute walk distance after critical illness: A systematic review and metaanalysis. J Intensive Care Med 2021;36:343-51.

31 Skinner EH, Haines KJ, Berney S, et al. Usual care physio- 
therapy during acute hospitalization in subjects admitted to the ICU: An observational cohort study. Respir Care 2015;60:1476-85.

32 Belli S, Balbi B, Prince I, et al. Low physical functioning and impaired performance of activities of daily life in COVID-19 patients who survived the hospitalisation. Eur Respir J 2020;56:2002096.

33 Shimogai T, Izawa KP, Kawada M, Kuriyama A. Factors affecting discharge to home of medical patients treated in an intensive care unit. Int J Environ Res Public Health 2019;16:4324. 